\title{
Avian assemblages at Paya Indah Natural Wetland Reserve, Malaysia
}

\begin{abstract}
Estimating accurate avian assemblages such as density and diversity are key factors for the conservation and management of birds and their habitats. Avian assemblages based on status in five habitats at Paya Indah Natural Wetland Reserve were determined using the distance sampling, point count method, at 121 count stations. A total of 100 bird species (64 resident, 18 migratory, 16 resident-migratory and 2 vagrant bird species) were detected from November 2012 to January 2014. The highest bird density was recorded for resident birds (72.2 \pm 3.8 bird's ha-1) and the lowest for vagrant birds $(0.1 \pm 0.1$ birds ha- 1$)$. In which Resident birds have significantly higher density, species diversity, richness and evenness compared to resident-migrant and migratory birds.
\end{abstract}

Keyword: Avifauna; Density; Distance sampling; Diversity; Wetland 